\title{
Three-dimensional maps of interstellar dust in the Local Arm: using Gaia, 2MASS, and APOGEE-DR14
} \author{
F. Arenou ${ }^{1}$, and N. Leclerc ${ }^{1}$ \\ 1 GEPI, Observatoire de Paris, PSL University, CNRS, 5 Place Jules Janssen, 92190 Meudon, France \\ e-mail: rosine. lallement@obspm. fr \\ 2 Irfu/DAp, CEA, Université Paris-Saclay, 91190 Gif-sur Yvette, France \\ 3 Université Paris Diderot, AIM, Sorbonne Paris Cité, CEA, CNRS, 91191 Gif-sur-Yvette, France \\ 4 Université Grenoble Alpes, CNRS, IPAG, 38000 Grenoble, France \\ 5 ACRI-ST, Sofia-Antipolis, France
}

R. Lallement ${ }^{1}$, L. Capitanio ${ }^{1}$, L. Ruiz-Dern ${ }^{1}$, C. Danielski ${ }^{1,2,3}$, C. Babusiaux ${ }^{4,1}$, J. L. Vergely ${ }^{5}$, M. Elyajouri ${ }^{1}$,

Received 14 February 2018 / Accepted 16 April 2018

\begin{abstract}
Context. Gaia data and stellar surveys open the way to the construction of detailed 3D maps of the Galactic interstellar (IS) dust based on the synthesis of star distances and extinctions. Dust maps are tools of broad use, also for Gaia-related Milky Way studies. Aims. Reliable extinction measurements require very accurate photometric calibrations. We show the first step of an iterative process linking 3D dust maps and photometric calibrations, and improving them simultaneously.

Methods. Our previous 3D map of nearby IS dust was used to select low-reddening SDSS/APOGEE-DR14 red giants, and this database served for an empirical effective temperature- and metallicity-dependent photometric calibration in the Gaia G and 2MASS $K_{s}$ bands. This calibration has been combined with Gaia $G$-band empirical extinction coefficients recently published, $G, J$, and $K_{s}$ photometry and APOGEE atmospheric parameters to derive the extinction of a large fraction of the survey targets. Distances were estimated independently using isochrones and the magnitude-independent extinction $K_{J-K_{S}}$. This new dataset has been merged with the one used for the earlier version of dust map. A new Bayesian inversion of distance-extinction pairs has been performed to produce an updated 3D map.

Results. We present several properties of the new map. A comparison with 2D dust emission reveals that all large dust shells seen in emission at middle and high latitudes are closer than $300 \mathrm{pc}$. The updated distribution constrains the well-debated, X-ray bright North Polar Spur to originate beyond $800 \mathrm{pc}$. We use the Orion region to illustrate additional details and distant clouds. On the large scale the map reveals a complex structure of the Local Arm. Chains of clouds of $2-3 \mathrm{kpc}$ in length appear in planes tilted by $\simeq 15^{\circ}$ with respect to the Galactic plane. A series of cavities oriented along a $1 \simeq 60-240^{\circ}$ axis crosses the Arm.

Conclusions. The results illustrate the ongoing synergy between 3D mapping of IS dust and stellar calibrations in the context of Gaia. Dust maps provide prior foregrounds for future calibrations appropriate to different target characteristics or ranges of extinction, allowing us in turn to increase extinction data and produce more detailed and extended maps.
\end{abstract}

Key words. local insterstellar matter - ISM: structure - dust, extinction - Galaxy: structure

\section{Introduction}

Three-dimensional (3D) maps of the Galactic interstellar matter (ISM) are a general tool for various purposes: studies of foreground, background, or environment for specific Galactic objects, modeling of photon or high-energy particle propagation, estimates of integrated Galactic foregrounds, and finally - of particular importance today in the context of Gaia - corrections for the reddening of the observed Milky Way stars. The construction of 3D maps requires massive amounts of distance-limited interstellar (IS) absorption data, i.e., they are necessarily based on absorption by IS dust or gas in the light of stellar objects, and the corresponding distances to the targets.

The synthesis of distance-limited absorption data to retrieve $3 \mathrm{D}$ information on the IS matter was done in two main ways. The first method distributes the data into solid angles and treats each radial direction independently. Following this method, Bayesian derivations of color excess radial profiles have been performed along radial directions, sightline by sightline. The first map was produced by Arenou et al. (1992) based on HIPPARCOS data.
Much later, the first map based on a massive survey from the ground was prepared by Marshall et al. (2006) who used 2MASS photometry and the Besançon model of stellar population synthesis (Robin et al. 2012b) to produce extinction curves at low Galactic latitudes $\left(-10 \leq \mathrm{b} \leq+10^{\circ}\right)$, and, from their derivatives, $3 \mathrm{D}$ maps of the IS dust up to $8 \mathrm{kpc}$ in the Galactic center hemisphere. Chen et al. (2013), and then Schultheis et al. (2014a), used the Besançon model and the VVV survey to produce 2D and 3D maps of the Bulge. Sale et al. (2014) used photometric data from the IPHAS Survey to reconstruct extinction radial profiles and dust maps at a better resolution for $-5 \leq b \leq+5^{\circ}$ and up to $5 \mathrm{kpc}$. Green et al. (2015) used 2MASS and PanSTARRS-1 data for 800000 stars and color-color diagrams to derive reddening radial profiles at very high angular resolution (on the order of $7^{\prime}$ ) in the whole region of the sky accessible for these two surveys. In parallel, maps of the Local Interstellar Bubble were drawn by van Loon et al. (1982) based on neutral sodium and diffuse interstellar band (DIB) data, and large-scale maps of the integrated IS matter based on massive amounts of DIB 
measurements have also been produced by Kos et al. (2014) and Zasowski et al. (2015).

Full 3D inversions differ from the previous technique by imposing spatial correlations between volume densities of IS matter in all directions, therefore linking adjacent sightlines. Three-dimensional inversions following a technique developed by Vergely et al. (2001) and with mono-modal and then bimodal covariance functions have been applied to the nearby ISM and much smaller datasets than in the case of the works cited above: columns of gaseous species (Welsh et al. 2010), color excesses from ground-based photometry (Vergely et al. 2010; Lallement et al. 2014), or composite sources combining photometry and DIBs (Capitanio et al. 2017). Sale \& Magorrian (2014, 2015, 2018) developed a Gaussian field method adapted to realistic multi-scale IS matter distributions. Rezaei Kh. et al. (2017) also developed a 3D inversion method and tested their new technique on extinction-distance measurements derived from the APOKASC catalog of Rodrigues et al. (2014).

The astrometric ESA mission Gaia (Gaia Collaboration 2016a) is an unprecedented opportunity to build improved 3D maps of the Galactic ISM. Parallax distances for more than a billion stars (Gaia Collaboration 2016b) will become available for combination with all types of distance-limited absorption data. Gaia will additionally provide extremely precise photometric measurements and in turn new extinction estimates, either from the satellite data only or in combination with ground-based stellar photometric or spectro-photometric surveys (Gaia Collaboration 2016a). Extinction estimates based on Gaia include wide-band photometry only, i.e., integrated magnitudes in the $G$-band and blue and red photometer (BP and RP) passbands, spectro-photometric determinations additionally based on the two BP and RP spectra, and in future more accurate determinations based on BP, RP, $G$, as well as stellar parameters from the Radial Velocity Spectrometer (RVS) spectra are expected.

Radial Velocity Spectrometer measurements will be restricted to bright targets. Prior to the release of the RVS spectra, and in general for all stars too faint to be characterized by the spectrograph, extinction estimates based on Gaia low-resolution data will suffer from the degeneracy between extinction and spectral energy distribution (Liu et al. 2012). This is why calibrations of the photometric bands and extinction coefficients as a function of the stellar parameters and the absorption are needed to properly determine the interstellar reddening and exploit Gaia data in conjunction with spectroscopic surveys, either for stellar science purposes or interstellar medium studies.

Three-dimensional maps of the nearby ISM may be used to select non-reddened or weakly reddened targets to enter empirical photometric calibrations, or more generally as a source of prior solutions for the extinction to be used in additional calibrations. Reciprocally, extinction measurements using the new calibrations can feed the distance-extinction databases, thus allowing the construction of improved and more extended maps. In this work we describe the first step of this iterative approach based on stellar - interstellar synergy. The 3D maps of Capitanio et al. (2017) based on wide- and narrowband photometry were used by Ruiz-Dern et al. (2018) to calibrate the bright Tycho-Gaia Astrometric Solution (TGAS) subset of Gaia Red Clump in the Gaia $G$ band, including temperature-metallicity-color relations using APOGEE-DR13 (Albareti et al. 2017) stellar parameters. They similarly served to select low-reddening targets to enter calculations of effective temperature and absorption-dependent coefficients in the $G$ band (Danielski et al. 2018). As part of the present work, the Ruiz-Dern et al. (2018) calibration was updated to the

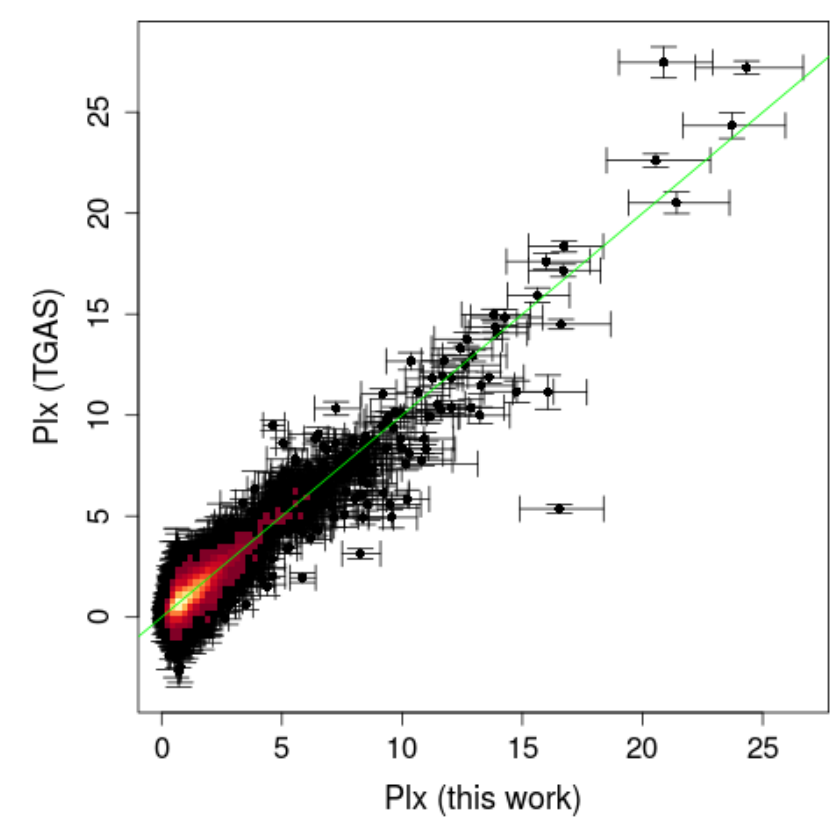

Fig. 1. Comparison of photometric distances obtained in this work with the Gaia/TGAS parallax distances for 13451 APOGEE stars. The color scale represents the square root of the density of stars, with yellow (resp. black) for the highest (resp. lowest) density.

APOGEE-DR14 stellar parameters (Abolfathi et al. 2018). This extended empirical calibration and the Danielski et al. (2018) extinction coefficients were combined with the APOGEE-DR14 stellar parameters and with 2MASS (Skrutskie et al. 2006) and Gaia $G$-band photometric data to derive extinctions for a large fraction of the targets. Distances to the targets were independently estimated based on isochrones. A new 3D inversion of distance-extinction pairs was subsequently performed after inclusion of this new dataset in the previous catalog.

In Sect. 2 we describe the method used to estimate the distances to the APOGEE targets and how the Gaia red giant calibrations performed by Ruiz-Dern et al. (2018) were updated to parameters of the APOGEE data release 14. We describe how this calibration and Gaia-G band extinction coefficients were used together with 2MASS and Gaia-G band data and with APOGEE atmospheric parameters to derive individual extinctions for those targets. In Sect. 3 we describe the resulting additional database and the new 3D inversion. We compare 2D maps of cumulative reddening up to $300 \mathrm{pc}, 800 \mathrm{pc}$ and finally up to the boundaries of the computational volume with dust emission maps. The comparison shows which of the main features seen in emission have been assigned a location. We illustrate the dust map improvements, present some new results and new online tools to use the maps in Sect. 4. In Sect. 5 we describe how the new augmented extinction-distance database has served to add local details to the dust distribution used in the Besançon model to enter the Gaia Universe Model Snapshot (GUMS, Robin et al. 2012a). We conclude and discuss future 3D map improvements in Sect. 6.

\section{Distance-extinction measurements of APOGEE-DR14 targets}

To derive the distance and extinction of the APOGEE objects, we use photometric data from both Gaia DR1 and 2MASS, and spectroscopic parameters such as effective temperature $T_{\text {eff }}$, 
Table 1. Updated coefficients and range of applicability of the $\hat{T}$ vs. $\left(G-K_{s}\right)_{0}$ relation (top table) and the $\hat{T}$ vs. $\left(G-K_{s}\right)_{0}$ relation (bottom table), $Y=a_{0}+a_{1} X+a_{2} X^{2}+a_{3}[\mathrm{Fe} / \mathrm{H}]+a_{4}[\mathrm{Fe} / \mathrm{H}]^{2}+a_{5} X[\mathrm{Fe} / \mathrm{H}]$, where $X$ and $Y$ are either the $\left(G-K_{s}\right)_{0}$ color or the normalized effective temperature $\hat{T}=T_{\text {eff }} / 5040$.

\begin{tabular}{|c|c|c|c|c|c|c|c|c|c|c|c|}
\hline$T_{\text {eff }}$ & $\left(G-K_{s}\right)_{0}$ range & {$[\mathrm{Fe} / \mathrm{H}]$ range } & $a_{0}$ & $a_{1}$ & $a_{2}$ & $a_{3}$ & $a_{4}$ & $a_{5}$ & $\mathrm{RMS}_{\left[T_{\mathrm{eff}}(K)\right]}$ & $\%_{\text {outliers }}$ & $\mathrm{N}$ \\
\hline$\hat{T}$ & {$[1.6,3.4]$} & {$[-2.3,0.4]$} & $1.591 \pm 0.021$ & $-0.413 \pm 0.018$ & $0.048 \pm 0.004$ & $0.009 \pm 0.001$ & - & - & 47.7 & $0.2 \quad 8$ & 855 \\
\hline Color & $T_{\text {eff }}$ range $(\mathrm{K})$ & {$[\mathrm{Fe} / \mathrm{H}]$ range } & $a_{0}$ & $a_{1}$ & $a_{2}$ & $a_{3}$ & $a_{4}$ & $a_{5}$ & $\operatorname{RMS}_{\left[G-K_{s}\right]}$ & $\%_{\text {outliers }}$ & $\mathrm{N}$ \\
\hline$\left(G-K_{s}\right)_{0}$ & {$[3761,5288]$} & {$[-2.3,0.4]$} & $12.884 \pm 0.423$ & $-18.592 \pm 0.886$ & $7.554 \pm 0.464$ & $-0.080 \pm 0.115$ & $0.022 \pm 0.008$ & $0.158 \pm 0.121$ & 0.04 & 0.28 & 855 \\
\hline
\end{tabular}

Notes. The range of temperatures of the $\left[\hat{T},\left(G-K_{s}\right)_{0}\right]$ calibration (second table) is given in $T_{\text {eff }}($ not $\hat{T})$.

metallicity $[\mathrm{Fe} / \mathrm{H}]$, and surface gravity $\log (g)$ from APOGEEDR14 (Abolfathi et al. 2018; Majewski et al. 2017). Here we used the ASPCAP parameters (García Pérez 2016). The crossmatch between the catalogs was done using the 2MASS ID provided in APOGEE and the 2MASS-GDR1 cross-matched catalog of Marrese et al. (2017).

\subsection{Distance}

We computed the distance modulus of 161683 APOGEE stars by using a Bayesian method on the Padova isochrones (Bressan et al. 2012, CMD2.7) and the magnitude independent of extinction $K_{J-K_{s}}$. We adopted the Initial Mass Function (IMF) of Chabrier (2001) on the mass distribution prior, and selected a flat distribution for the prior on age. A criterion of $\chi_{0.99}^{2}$ was applied to reject stars that are too far from the isochrones.

The results obtained were compared to the four distance estimations provided directly by the APOGEE team ${ }^{1}$ : BPG (at 50 percentile, Santiago et al. 2016; Queiroz et al. 2018), NAOC (Wang et al. 2016), NICE (Schultheis et al. 2014b), and NMSU (Holtzman et al., in prep.). They all rely upon the calibrated stellar atmospheric parameters of APOGEE-DR14 and isochrones. The results, as expected, agree, with small differences especially at the greatest distances. The greatest differences are with the NICE estimations, with only $0.9 \%$ of outliers at $5 \sigma$.

We also compared our distances to the Gaia parallaxes for a subset of 13451 APOGEE stars present in the TGAS catalog (Fig. 1). We find only $0.1 \%$ of outliers and the normalized residuals follow a normal distribution centered around zero if we account for the small Gaia parallax zero point offset of -0.04 mas (Arenou et al. 2017).

\subsection{Extinction}

As in the analysis of Ruiz-Dern et al. (2018), made for Red Clump stars, we used the Capitanio et al. (2017) 3D maps to select those APOGEE red giant targets for which sightlines are devoid of clouds and extinction is expected to be very weak. We used those targets to update the coefficients of the effective temperature metallicity-dependent calibrations $\hat{T}$ versus $\left(G-K_{s}\right)_{0}$ and $\left(G-K_{s}\right)_{0}$ versus $\hat{T}$, where $\hat{T}=T_{\text {eff }} / 5040 \mathrm{~K}$, using the last APOGEE-DR14 catalog. The new results are shown in Table 1 , together with the respective intervals of validity (for the formalism, see Ruiz-Dern et al. 2018).

In the second stage, with the purpose of determining individual extinctions, we selected from the APOGEE-2MASSGaia cross-matched catalog those stars with effective tempera-

\footnotetext{
1 http://wWw.sdss.org/dr14/data_

access/value-added-catalogs/?vac_id=

apogee-dr14-based-distance-estimations/
}

ture $3761 \mathrm{~K}<T_{\text {eff }} \pm \sigma_{T_{\text {eff }}}<5288 \mathrm{~K}$ and metallicity -2.3 dex $<$ $[\mathrm{Fe} / \mathrm{H}]<0.42$ dex in order to work within the same limits of the photometric calibration (Table 1). We removed all those objects whose temperature error $\sigma_{T_{\text {eff }}}$ or metallicity error $\sigma_{[\mathrm{Fe} / \mathrm{H}]}$ were unknown. Finally, we discarded stars with photometric errors $\sigma_{G}, \sigma_{K_{s}}, \sigma_{J}>0.05 \mathrm{mag}$. The application of these criteria delivered a sample of stars.

We used a Markov chain Monte Carlo (MCMC, Brooks et al. 2012) method similar to that used in Danielski et al. (2018) to properly account for errors. The MCMC used the jags algorithm (Plummer 2003) encompassed in the runjags ${ }^{2}$ library for the $\mathrm{R}$ program language.

For each star in the sample we set effective temperature and metallicity to follow the normal distribution $\mathcal{N}$ such as $T_{\text {eff }}^{\prime} \sim$ $\mathcal{N}\left(T_{\text {eff }}, \sigma_{\mathrm{T}_{\text {eff }}}^{2}\right)$ and $[\mathrm{Fe} / \mathrm{H}]^{\prime} \sim \mathcal{N}\left([\mathrm{Fe} / \mathrm{H}], \sigma_{[\mathrm{Fe} / \mathrm{H}]}^{2}\right)$, where $T_{\text {eff }}$ and $[\mathrm{Fe} / \mathrm{H}]$ are the observed temperature and metallicity, and $\sigma_{\text {Teff }}^{2}$ and $\sigma_{[\mathrm{Fe} / \mathrm{H}]}^{2}$ are the respective observed variances. Consequently, $\hat{T}^{\prime}$ was defined as $\hat{T}^{\prime}=T_{\text {eff }}^{\prime} / 5040$.

We measured the intrinsic colors $\left(G-K_{s}\right)_{0}$ and $\left(J-K_{s}\right)_{0}$ using the photometric calibration relations: $\left(G-K_{s}\right)_{0}$ versus $\hat{T}^{\prime}$ (Table 1$)$ and $\left(J-K_{s}\right)_{0}$ versus $\left(G-K_{s}\right)_{0}$ provided by Ruiz-Dern et al. (2018).

The likelihood is expressed using the star observed colors $\left(G-K_{s}\right)_{\mathrm{obs}}$ and $\left(J-K_{s}\right)_{\mathrm{obs}}$,

$$
\begin{aligned}
& \left(G-K_{s}\right)_{\mathrm{obs}} \sim \mathcal{N}\left(\left(G-K_{s}\right)_{0}+\left(k_{G}-k_{K_{s}}\right) \cdot A_{0}, \sigma_{G-K_{s}}^{2}\right), \\
& \left(J-K_{s}\right)_{\mathrm{obs}} \sim \mathcal{N}\left(\left(J-K_{s}\right)_{0}+\left(k_{J}-k_{K_{s}}\right) \cdot A_{0}, \sigma_{J-K_{s}}^{2}\right),
\end{aligned}
$$

where $\sigma_{G-K_{s}}^{2}=\left(\sigma_{G}^{2}+\sigma_{K_{s}}^{2}\right)$ and $\sigma_{J-K_{s}}^{2}=\left(\sigma_{J}^{2}+\sigma_{K_{s}}^{2}\right)$ and where $k_{G}, k_{J}$, and $k_{K_{s}}$ are the extinction coefficients for the Gaia $G$ band and 2MASS $J$ and $K_{s}$ bands, respectively. These coefficients are expressed as a function of the color $\left(G-K_{s}\right)_{0}$ and extinction $A_{0}$, as provided by Danielski et al. (2018). Finally, we set the extinction $A_{0}$ as the only free parameter allowed to vary following a uniform prior distribution $\mathcal{U}$ between 0 and 20 mag: $A_{0} \sim \mathcal{U}(0,20)$.

Each MCMC was run using $10^{4}$ steps and a burn-in of 4000 . For each star we obtained its extinction mean value and standard deviation. We tested the validity of the results through a $\chi_{0.99}^{2}$ criterion and we rejected those stars whose individual extinction did not comply with this criterion or whose value was not within the validity ranges of the $k_{G}$ extinction coefficient. The resulting sample of valid objects consists of 148744 stars.

Extinctions are provided together with distances for the BPG, NAOC, and NICE methods, providing respectively $A_{V}$, $A_{K}$, and $E(J-K)$. We present in Fig. 2 the comparison with those studies that agree reasonably well overall considering the different approaches.

\footnotetext{
2 https://cran.r-project.org/web/packages/runjags/ runjags.pdf
} 

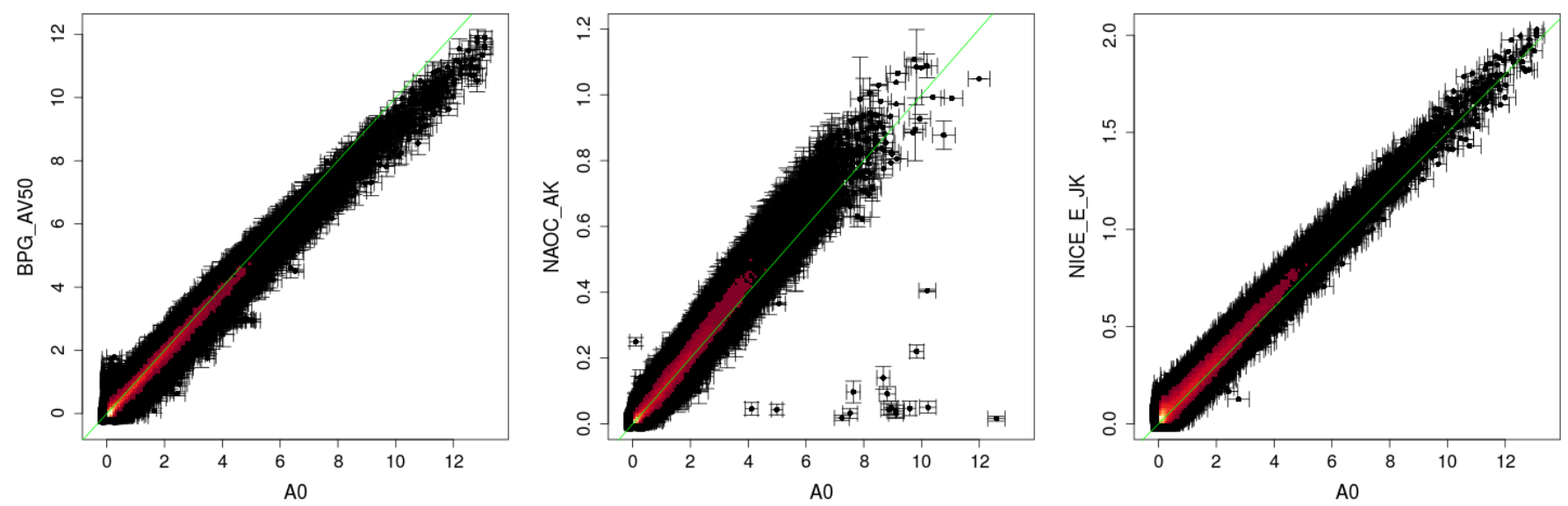

Fig. 2. Comparison between the extinction $A_{0}$ derived here with (from left to right) BPG $A_{V}$ (red line slope: 1.0), NAOC $A_{K}$ (red line slope: 0.1 ), and NICE $E(J-K)$ (red line slope: 0.15 ). The color scale represents the square root of the density of stars, with yellow (resp. black) for the highest (resp. lowest) density.

\section{Construction of updated 3D maps}

\subsection{Data selection and method}

As described in Sect. 2, distance-extinction pairs and corresponding uncertainties have been obtained for $\simeq 149000$ APOGEE-DR14 targets. As in the case of the previously inverted distance-extinction database, we retained for the inversion all targets for which the estimated distance is smaller than $2.6 \mathrm{kpc}$, the distance to Plane $\mathrm{Z}$ smaller than $600 \mathrm{pc}$, and the estimated relative error on the distance smaller than $30 \%$. For the 42633 selected targets following these criteria, extinctions at $550 \mathrm{~nm} \mathrm{~A}_{0}$ were converted into color excess $E(B-V)$ assuming $\mathrm{A}_{0}=3.1 E(B-V)$. We note that, for this reason, in the following description of the inversion and maps we use both terms extinction or color excess since they are simply proportional.

These new data were merged with those corresponding to the 30000 targets used by Capitanio et al. (2017). Doing so, we combined for the first time extinction measurements that were entirely extracted from ground-based photometry and new measurements using both ground- and Gaia space-based G-band photometry. We have kept in the former dataset the $\simeq 4900$ extinction estimates based on the $15273 \mu$ diffuse interstellar band extracted from the DR13 APOGEE spectra. These estimates were made for APOGEE telluric standard stars (TSSs) and do not overlap with the above targets that are science targets only. The TSSs are brighter and bluer stars and for these objects APOGEE model spectra are not appropriate; the DIBs were extracted following the technique developed by Elyajouri et al. (2016). We also added a few hundred extinctions estimated from uvby $\beta$ photometry by Kaltcheva et al. (2014) for targets in the direction of the supershell GSH 305+01-24 region. Their distance range from 0.1 to $2 \mathrm{kpc}$ is in good agreement with that of the APOGEE targets (see below). Figure 3 shows the locations of the projection onto the Plane of the initial (Capitanio et al. 2017) targets and all additional targets, in the two cases for targets with $|b| \leq 10^{\circ}$. At variance with the Capitanio et al. (2017) targets, most of which were located closer than $500 \mathrm{pc}$ and for a large fraction even closer within the Local Cavity, the new APOGEE science targets are distributed at greater distances and fill the space until $2.0 \mathrm{kpc}$. This is due to the use of the infrared spectral domain and the APOGEE sensitivity and illustrates the qualities of the survey. The complementarity of distances makes

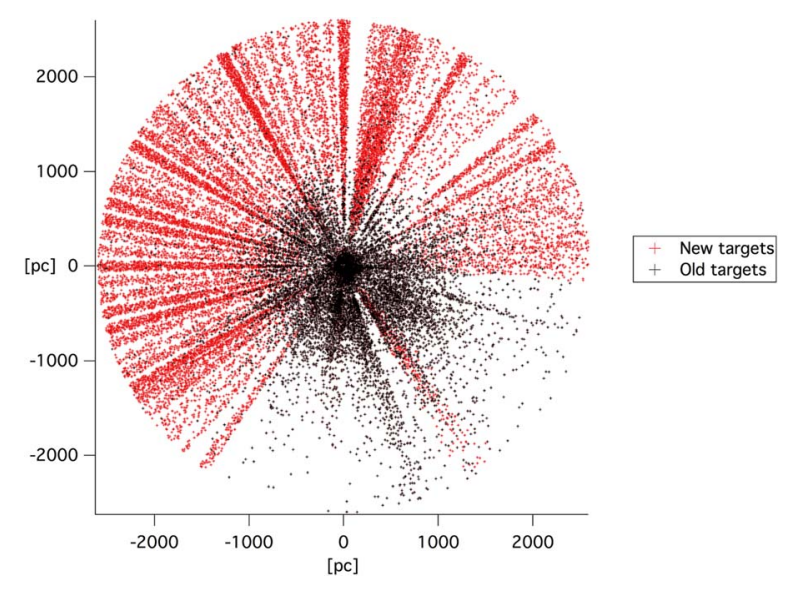

Fig. 3. Projections onto the Galactic plane of target stars entering the new inversion and with Galactic latitude $-10 \leq \mathrm{b} \leq+10^{\circ}$. The targets previously used by Capitanio et al. (2017) are the black dots. The additional targets are the red dots.

the merged catalog very appropriate for $3 \mathrm{D}$ mapping close to the Plane.

We performed a regularized Bayesian inversion of all individual color excesses, following the pioneering technique described in Tarantola \& Valette (1982) and successively applied to the local IS dust by Vergely et al. (2001, 2010), Lallement et al. (2014), and Capitanio et al. (2017). This inversion uses sightline-integrated data to create an analytic function of space coordinates that represents the local differential color excess or differential opacity at any point $P \delta(E(B-V)) / \delta d$, if $d$ is the distance along any sightline crossing $P$. This quantity, expressed in mag $\mathrm{pc}^{-1}$, is proportional to the volume density of absorbing dust or volume opacity. The inversion is a largely underconstrained problem, and to regularize it we impose a smoothing of the volume opacity: the absorbing dust must be distributed in volumes of an imposed minimum size and must have imposed maximum spatial gradients (or contrasts). The correlation function for the opacity in two points is the sum of the two kernels:

$\psi\left(x_{\mathrm{a}}, x_{\mathrm{b}}\right)=\frac{\sigma_{\chi_{0}}{ }^{2}}{\cosh \left(-\frac{\left|x_{\mathrm{a}}-\chi_{\mathrm{b}}\right|}{\chi_{0}}\right)}+\sigma_{\chi_{1}}{ }^{2} \exp \frac{-\left|x_{\mathrm{a}}-x_{\mathrm{b}}\right|^{2}}{\chi_{1}}$. 
In the present inversion the minimum sizes of the structures are $\chi_{0}=30 \mathrm{pc}$ and $\chi_{1}=15 \mathrm{pc}$, with the corresponding allowed contrasts $\sigma_{\chi_{0}}=0.8$ and $\sigma_{\chi_{1}}=1$.0. For more details on these choices see Lallement et al. (2014). Uncertainties on the color excess/extinction and on the target distance both enter the Bayesian adjustment. As stated above, input data have relative errors on distances lower then $33 \%$. There is no corresponding limitation for the color excess; however, there is a minimum imposed error of $0.01 \mathrm{mag}$. In case of the DIBs an additional uncertainty is introduced to represent the DIB/extinction ratio variability (Capitanio et al. 2017). As detailed in Lallement et al. (2014) the inversion algorithm combines the input errors on target distances and color excesses in a single quantity.

At variance with previous inversions and for computational reasons, the new 3D inversion was not done using all stellar targets simultaneously. The computation time varies as $\propto n_{\text {targets }}{ }^{2}$ if $n_{\text {targets }}$ is the number of targets entering the inversion. We chose to compute separately two maps based on targets with longitudes $l=\left(-95^{\circ}, 95^{\circ}\right)$ and $\left(85^{\circ},-85^{\circ}\right)$, i.e., roughly the Galactic center hemisphere and the Galactic anticenter hemisphere, respectively, with an overlap of $5^{\circ}$ on both extremities. The inversion for the center hemisphere was done with 35748 stellar targets and the anticenter with 38 806. In total 71357 sightlines were used, of which 3197 were in overlapping regions. As in Capitanio et al. (2017), a large-scale prior distribution based on Green et al. (2015) has been used for these two inversions. For each inversion we obtained a $3 \mathrm{D}$ distribution in a cube $[-2000,2000] \mathrm{pc} \times[-2000,2000] \mathrm{pc} \times[-300,300] \mathrm{pc}$ and this density is discretized in voxels of 5 pc per side, i.e., $5 \times 5 \times 5$. The merging of the two distributions in the overlapping volume is not a simple average, but instead a weighted average depending on the angular distance to the $b=90^{\circ}$ or $b=270^{\circ}$ limiting surfaces. Several checks were performed to ensure that the resulting distribution is very similar to that expected in a single inversion.

\subsection{Error estimates}

The resulting map of volume opacities has intrinsic errors that are due to the regularization because it tends to smooth the density structures and distribute the opacity in volumes that are wider than the actual values. Structures smaller than $\sim 20 \mathrm{pc}$, like dark clouds or small supernova remnant (SNR) cavities, cannot be resolved with the present dataset and algorithm. This also introduces errors on the cumulative color excess computed by integration through the 3D distribution. Uncertainties on cloud locations and opacities are dependent on the target volume density, the clumpiness of the structures and on uncertainties on distances and extinctions, i.e., they vary strongly within the computational volume. A precise error calculation for this type of Bayesian inversion method was proposed by Sale et al. (2014); however, it is difficult to implement and requires a very long computation time. We decided here to limit our error estimates to the integrated extinction between the Sun and each location in the computational volume. These error estimates can be obtained from the online tools described below. An estimate of the uncertainty on the distance to the near side of each structure is also given by the tool. The distance uncertainty was calculated in each point $(\mathrm{x}, \mathrm{y}, \mathrm{z})$ of the 3D [800] $\times[800] \times[300]$ voxel matrix (we recall that each voxel center corresponds to $5 \mathrm{pc}$ distant points in the $[-2000: 2000] \mathrm{pc} \times[-2000: 2000] \mathrm{pc} \times[-300: 300] \mathrm{pc}$ volume), following the method of Capitanio et al. (2017), i.e., considering that the uncertainty is mainly dependent on the density of targets that constrain the structures. At each point $(x, y, z)$ we computed the target density $N$ in [200] $\mathrm{pc} \times[200] \mathrm{pc} \times$ [200] pc cubes centered on the point, and the associated distance error was estimated as the inverse of this density: $200 \times$ $N^{-\frac{1}{3}}$. The error on the cumulative reddening was computed in a different way than in Capitanio et al. (2017). At each point $(x, y, z)$ we used the same set of targets used for the distance uncertainty, i.e., located within a 200 pc $\times 200$ pc $\times 200$ pc cube around the point, and computed for these targets the mean value of the difference between the measured color excess (the input value) and that obtained by integration of the final adjusted differential color excess distribution between the Sun and the target. At large distances where data are scarce, this method results in very large errors. Since a limitation on the color excess is provided by 2D maps of integrated reddening based on dust emission, we used this limit as an upper limit on the integrated reddening between the Sun and any point whatever its distance. Doing so, we allowed for asymmetric errors and computed independently the negative and positive error intervals. For the upper limit on the integrated reddening we used the 2D maps of Schlegel et al. (1998, hereafter SFD98). Due to our smoothing constraints the SFD98 map is more appropriate than more recent high-resolution maps. At each point we computed the difference between the SFD98 value and the map-integrated reddening and compared this difference with the error calculated following the neighboring targets method previously described. We defined the positive error as the smallest of these two numbers. As a result of this choice, in some very rare cases where our integrated color excess is higher then the SFD98 value, the positive error becomes null. On the other hand, because the cumulative reddening must be positive, the negative error is chosen as the smallest of the two quantities: our computed error or the reddening itself.

\subsection{Inversion results: cumulative reddening maps}

Figures 4-6 show 2D maps of the cumulative color excess from the Sun to $300 \mathrm{pc}, 800 \mathrm{pc}$ and the boundaries of the computational volume, respectively. These cumulative color excess maps were simply computed by integrating the local differential color excess resulting from the inversion up to each chosen distance, for a grid of radial directions separated by $1^{\circ}$ in Galactic longitude and latitude. Superimposed are a few selected isocontours of the total Galactic reddening computed by Schlegel et al. (1998). The comparisons between the maps and the total SFD98 reddening do not allow us to estimate errors in our mapping, instead they allow us to disentangle structures that have been assigned a location in the 3D map within the chosen distance from structures that are located farther away.

The 300 pc-integrated maps are of particular interest. In the northern hemisphere, they clearly show strong coincidences between the SFD98 isocontour at $E(B-V)=0.025 \mathrm{mag}$ and the directions where the cumulative reddening reaches this value (see the colors and color-coding in Fig. 4). This implies that all of the northern high-latitude structures seen in the maps and characterized by $E(B-V)$ values on the order of few hundreds of a magnitude are closer than $300 \mathrm{pc}$. This was already perceptible in our previous maps (see Fig. 11 in Lallement et al. 2014), but is seen in a much clearer way here thanks to the addition of high-latitude APOGEE targets. On the contrary, in the southern hemisphere the same SFD98 isocontour at $0.025 \mathrm{mag}$ falls in directions for which the $300 \mathrm{pc}$ integrated reddening is weaker (darker blue color), suggesting that either a significant fraction of the emitting dust 

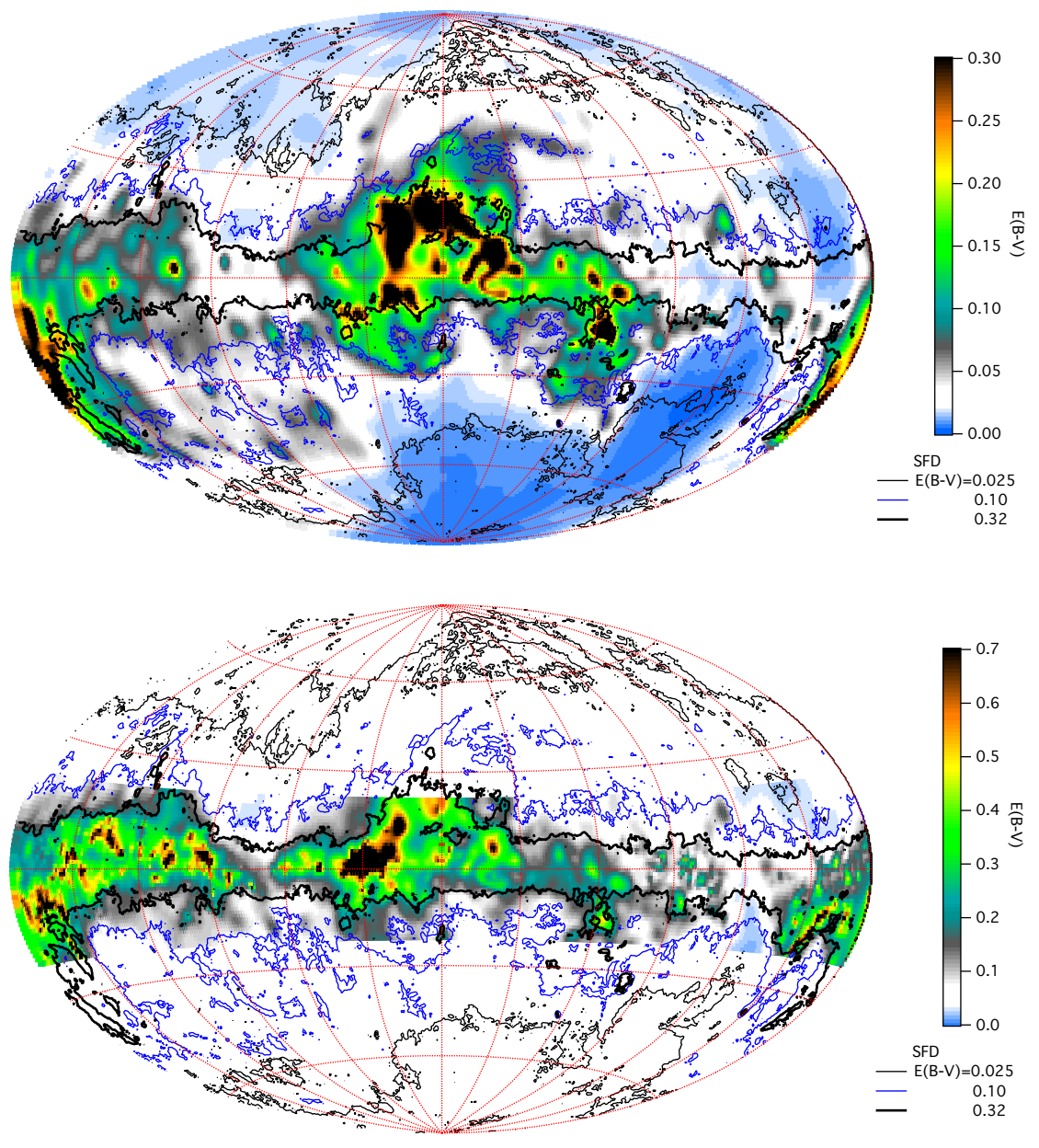

Fig. 4. Cumulative color excess $E(B-V)$ computed by integration of the differential color excess along radial directions, from the Sun up to a distance of $300 \mathrm{pc}$. Isocontours derived from the SFD 98 reddening map are superimposed. The comparison shows that all northern high-latitude arches seen at longitudes $-150 \leq 1 \leq+40^{\circ}$ and with $E(B-V) \geq$ $0.025 \mathrm{mag}$ are closer than $300 \mathrm{pc}$ (see text).

Fig. 5. Same as Fig. 4, for sightlines 800 pc long. Grid points are left blank at high Galactic latitudes (abs $(b)>\simeq 25^{\circ}$ ); for such sightlines $800 \mathrm{pc}$ distant sightline extremities are out of our $4000 \times 4000 \times$ $600 \mathrm{pc}^{3}$ computational volume. In the second quad$\operatorname{rant}\left(90 \leq 1 \leq 180^{\circ}\right)$ there is good agreement between SFD98 isocontours for $E(B-V)=0.32 \mathrm{mag}$ and locations corresponding to about the same value of our integrated color excess. This shows that most of the structures in these areas are within $800 \mathrm{pc}$. On the contrary, for a large fraction of the third and fourth quadrants $\left(180 \leq 1 \leq 270^{\circ}\right.$ and $270 \leq 1 \leq 360^{\circ}$, respectively) most of the dust seen in emission is beyond $800 \mathrm{pc}$.

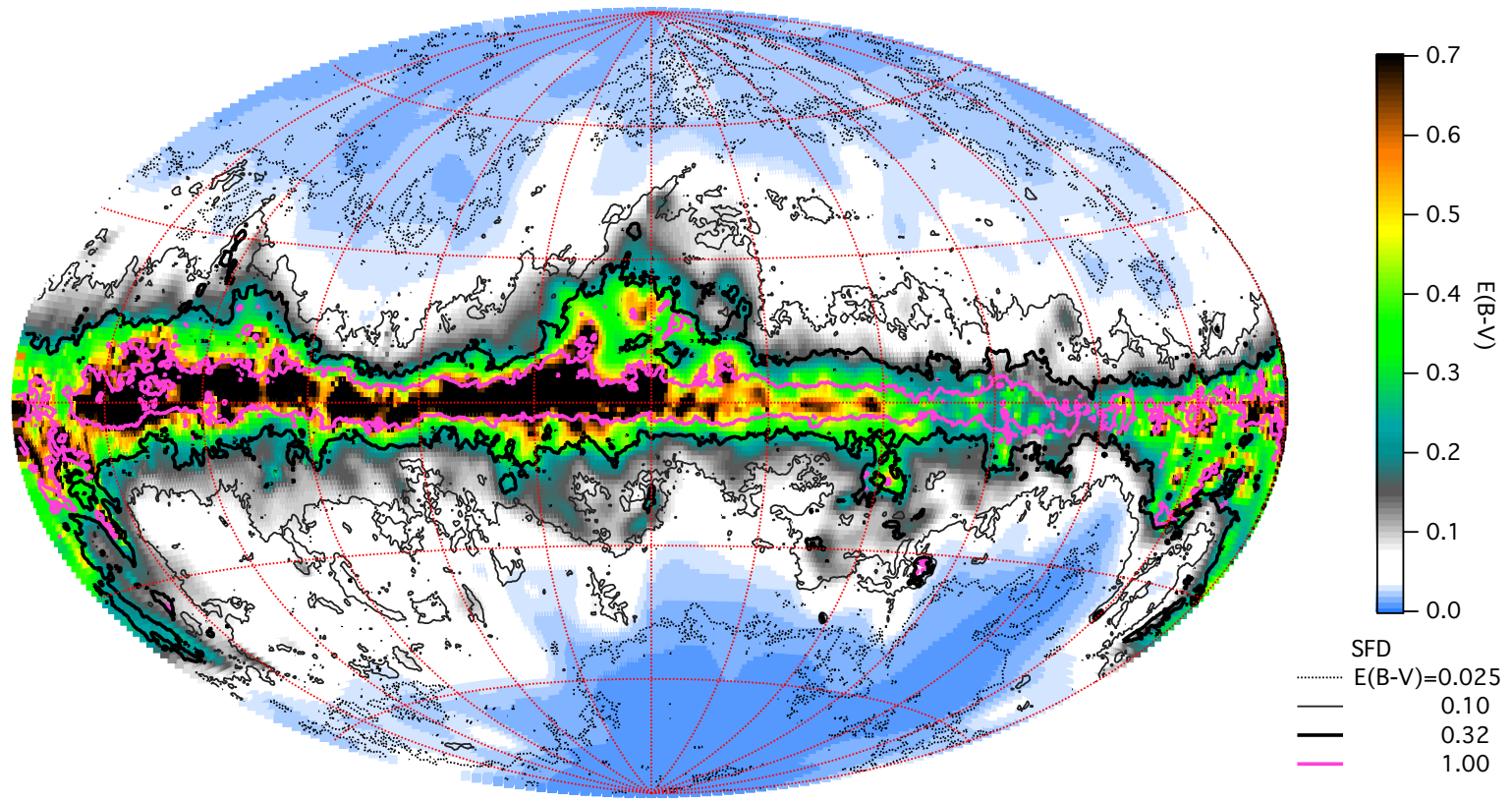

Fig. 6. Integrated color excess reached at the boundary of the computational volume. Isocontours derived from the SFD98 reddening map are superimposed.

is beyond this distance or the dust properties are different. This is particularly marked at longitudes centered around $l=$ $240^{\circ}$, i.e., towards the conspicuous Canis Major cavity below the Plane that is known to be filled with a large quantity of ionized gas and to be characterized by a low dust-to-gas ratio (Lallement et al. 2015). 


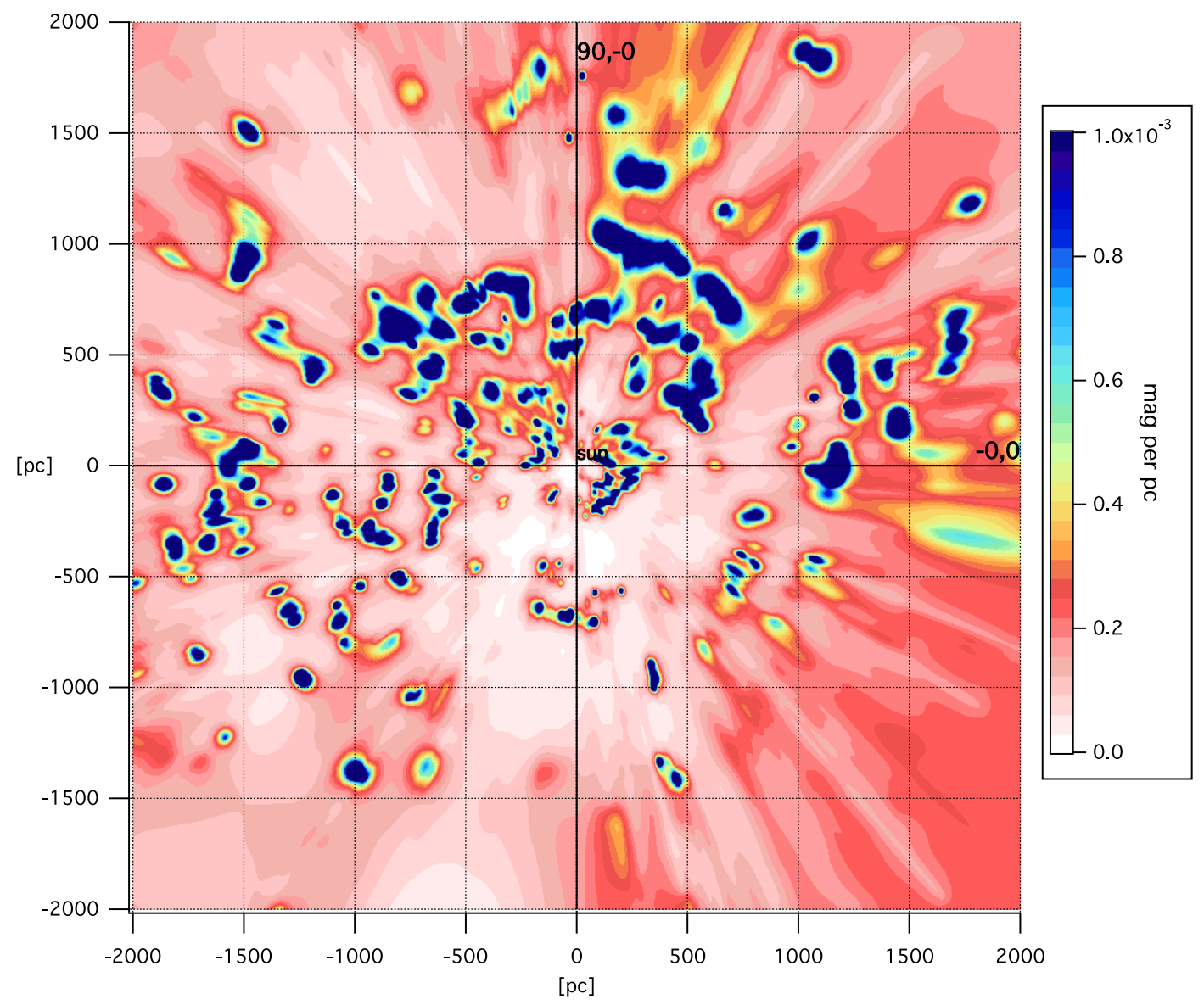

Fig. 7. Differential color excess in the Galactic plane computed as a planar cut along the Galactic equator through the 3D distribution. The Sun is at the center of the image (coordinates 0,0 ). The Galactic center direction is to the right. The units are mag pc ${ }^{-1}$. The map can be directly compared with the previous similar map from Capitanio et al. (2017).

\section{Three-dimensional dust maps and tools}

\subsection{Online tools}

The website dedicated to the use of the $3 \mathrm{D}$ maps ${ }^{3}$ has been updated. Three types of requests can be submitted. Tool (i) produces maps of the differential reddening or, equivalently, maps of the dust clouds for any planar cut through the computational volume. Differential reddening isocontours are added to help visualize the faint structures and cloud centers. A requested planar map is defined by the spherical coordinates of its center and the Galactic coordinates of the normal to the plane. Zooming in on the image is possible and images can be downloaded. Several examples of the use of this tool are given below. With tool (ii) the entire $3 \mathrm{D}$ distribution can be downloaded. This may be useful to improve computations of the energetic particle propagation and estimates of the radiation field, or for comparisons with evolutionary models of the ISM. With tool (iii) cumulative reddening curves with associated estimated uncertainties on the reddening and on the distances to the reddening jumps can be drawn on request for any direction and downloaded. This allows us to estimate the reddening at a given distance in a given direction.

Regarding those tools, we emphasize again that the main utility of our inverted maps is to provide the 3D localization and shape of the main nearby structures, i.e., the information contained in the 2D or 3D images representing the distribution (tools

\footnotetext{
3 http://stilism.obspm.fr
}

(i) and (ii)). The cumulative reddening curves (tool (iii)) provide the distances where reddening jumps occur, again information on the distances to the structures and their shapes; however, we caution that the absolute value of the reddening may be uncertain in clumpy regions due to the imposed redistribution of the absorbing matter in clouds of sizes larger than $15 \mathrm{pc}$. In such regions of the sky, tools based on more resolved maps (e.g., the Green et al. 2015 map) provide more reliable quantitative estimates.

\subsection{Dust distribution in various planes}

Figure 7 displays the differential color-excess in the Galactic plane. A comparison with the map based on our previous inversion (Fig. 5 in Capitanio et al. 2017) demonstrates that the addition of the APOGEE targets has resulted in significant mapping extension and in additional details. On the large scale, interestingly, the new structures that are now located beyond $1 \mathrm{kpc}$ in the first quadrant at longitudes between $1=0$ and $1=45^{\circ}$ appear as a prolongation of the series of clouds in the fourth quadrant at a distance of 500-1000 pc. Together, all these clouds seem to mark the forefront of the Sagittarius Arm. However, based on distributions outside the Plane, as discussed below, the boundary between the Local Arm and Carina-Sagittarius in the first quadrant is not as clearly defined as it may appear here in the Galactic plane.

Mapping improvements and extensions can be illustrated using cuts in the 3D distribution along vertical planes, and we show 


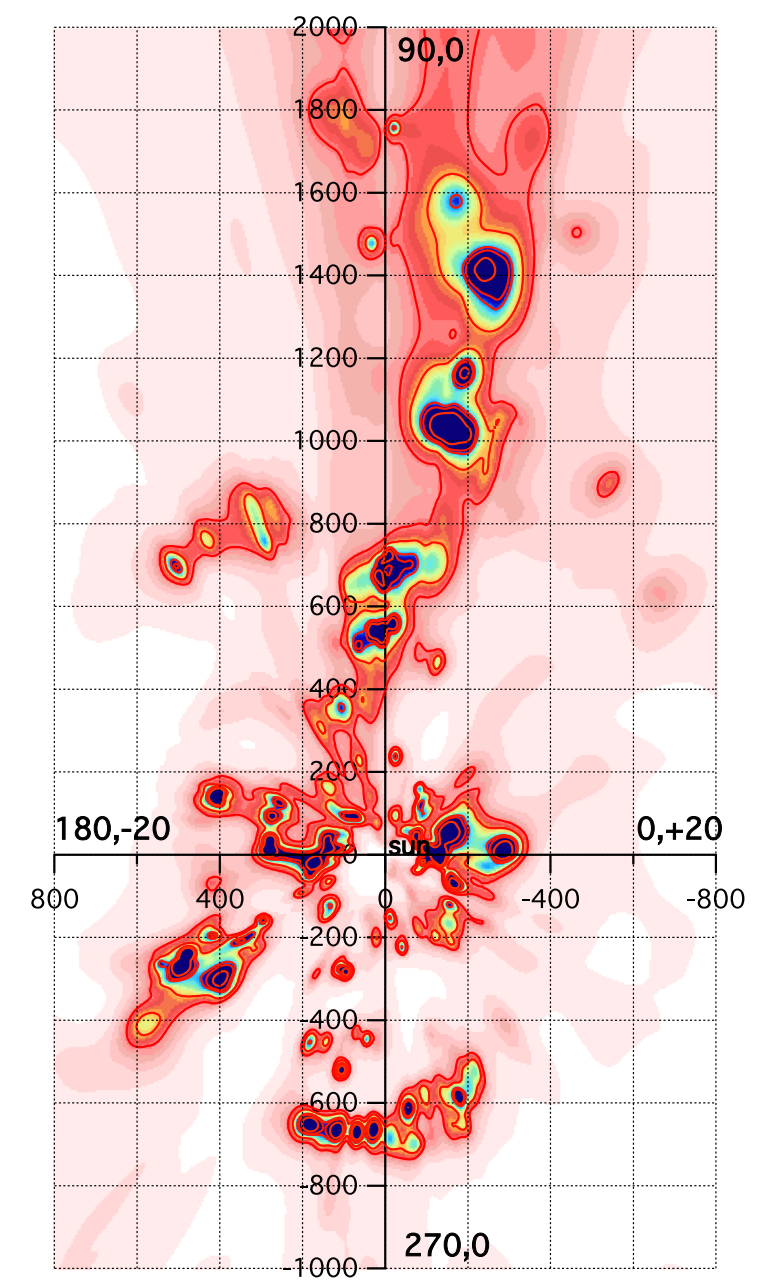

Fig. 8. Same as Fig. 7 in a Plane containing the Sun and inclined by $20^{\circ}$ with respect to the Galactic plane (ascending node along $(l$, b) $=\left(270^{\circ}, 0^{\circ}\right)$. The map reveals a $2 \mathrm{kpc}$ long conspicuous chain of cloud complexes. Iso-contours are for 0.0002, 0.00035, 0.00076, 0.001, $0.002 \mathrm{mag} \mathrm{pc}^{-1}$

here two regions of particular interest. Figure 9 corresponds to the vertical plane along the longitude $1=28^{\circ}$. This region has been investigated several times due to the presence of the conspicuous X-ray feature called the North Polar Spur that brightens above $\mathrm{b}=+9^{\circ}$ in the $20-30^{\circ}$ longitude range and whose origin is still a matter of lively debate (Planck Collaboration Int. XIX 2015; Sun et al. 2015; Sofue et al. 2016). According to most authors the X-ray emission originates very close to the Sun at $\sim 100 \mathrm{pc}$ in a nearby hot gas cavity blown by one or more supernovae, but its link with the Galactic center activity and more recently with the Fermi bubbles seen in gamma rays has been also claimed (see Lallement et al. 2016 for the various assumptions and references). Three-dimensional maps constitute a useful tool for locating the NPS source location. Puspitarini et al. (2014) searched for the potential cavity responsible for the X-rays, but could not find any nearby wide cavity that could be identified with the NPS. More recently Lallement et al. (2016) derived from dedicated XMM-Newton data that the NPS is absorption-bounded and not emissionbounded. The authors used 3D local and large-scale maps from 2MASS and PanSTARRS-1 (Green et al. 2015) to demonstrate that the NPS emission is absorbed by an opaque cloud located beyond at least $300 \mathrm{pc}$, and they found evidence for a much more distant origin. Figure 9 now reveals very clearly the massive absorbing structure that blocks the X-rays below $\sim b=9^{\circ}$ and shows that this absorbing cloud complex is wide and that its far side is at $\sim 800 \mathrm{pc}$. This provides new and strong evidence that the NPS source is beyond $800 \mathrm{pc}$, definitely outside the Local Arm. We believe that there is a fortuitous spatial coincidence between the extended northern HI arches seen with $21 \mathrm{~cm}$ data and the wide and conspicuous radio/X-ray NPS/Loop 1 structure extending to $\mathrm{b}=+75^{\circ}$. The former are local, as demonstrated by Puspitarini \& Lallement (2012) from absorption data for one of the shells and by the 300 pc-integrated emission map of Fig. 4 discussed in the previous section. The latter is generated much farther away.

Figure 10 illustrates the effects of the additional APOGEE targets in the region of the well-known Orion clouds. The top graph displays the new inverted dust distribution in a vertical plane along the longitude $1=212^{\circ}$. Superimposed are isocontours of the differential color excess from the Capitanio et al. (2017) distribution, i.e., without APOGEE targets. The comparison clearly reveals the addition of more distant clouds in the new maps, especially beyond $500 \mathrm{pc}$. At short distances there are no noticeable changes; however, in the intermediate distance range (between 400 and $600 \mathrm{pc}$ ), the Orion clouds display more substructures. Together, the three graphs in Fig. 10 show how the cloud distribution evolves with longitude, and reveal a very complex spatial distribution. We have compared these results with those from Schlafly et al. (2015), which are based on PanSTARRS photometry, and find full compatibility with the range of distances presented by the authors for the most important structures in their Figs. 2 and 4. We also tentatively located in Fig. 10 the structures identified by Lombardi et al. (2011) as A, B, N, as well as $\lambda$ Ori, Rosette, and Mon R2. For all of them there are clouds at distances and directions in very close agreement with those from Lombardi et al. (2011).

As an illustration of a plane that is not Sun-centered and has any orientation, we show in Fig. 11 the cloud distribution in the Gould belt plane as defined by Perrot \& Grenier (2003). The center of the image is $104 \mathrm{pc}$ from the Sun along the direction $(l, b)=\left(180.4^{\circ}, 0^{\circ}\right)$. The plane is inclined by $17.4^{\circ}$ with respect to the Galactic plane, and the ascending node is at the longitude $1=296^{\circ}$. It contains the Aquila rift clouds on the Galactic center hemisphere side, and Taurus and the chain of Orion clouds on the anticenter hemisphere side. We added the ellipse computed by Perrot \& Grenier (2003) as the closest solution for an expanding and rotating structure adjusted to the locations and motions of the main Gould belt structures. The figure strongly suggests that instead of a ring of clouds, the Gould belt is made of two distinct regions separated by a series of cavities and running parallel to this series (see Lallement et al. 2015 for a recent discussion of the Gould belt structure and origin). Figure 12 is similar to Fig. 11, the only difference being the longitude of the ascending node, now at $1=26^{\circ}$; in other words, this plane can be obtained by rotating the previous Gould belt plane by $90^{\circ}$ along a vertical $\left(\mathrm{b}=90^{\circ}\right)$ axis. The image in this plane shows clouds distributed along $\sim 4 \mathrm{kpc}$ along the $1 \simeq 35-225^{\circ}$ direction, far from the expected orientation of a Galactic Arm approximately aligned with the direction of rotation. This orientation is in better agreement with a bridge between Carina-Sagittarius and Perseus. For this reason, the name Orion bridge sometimes employed for the Local Arm seems more appropriate. Again, in this plane there seems to be a splitting of the whole structure into two main regions separated by a series of cavities, including the $150 \mathrm{pc}$ Local Cavity in the middle, and two very long and wide cavities towards longitudes 


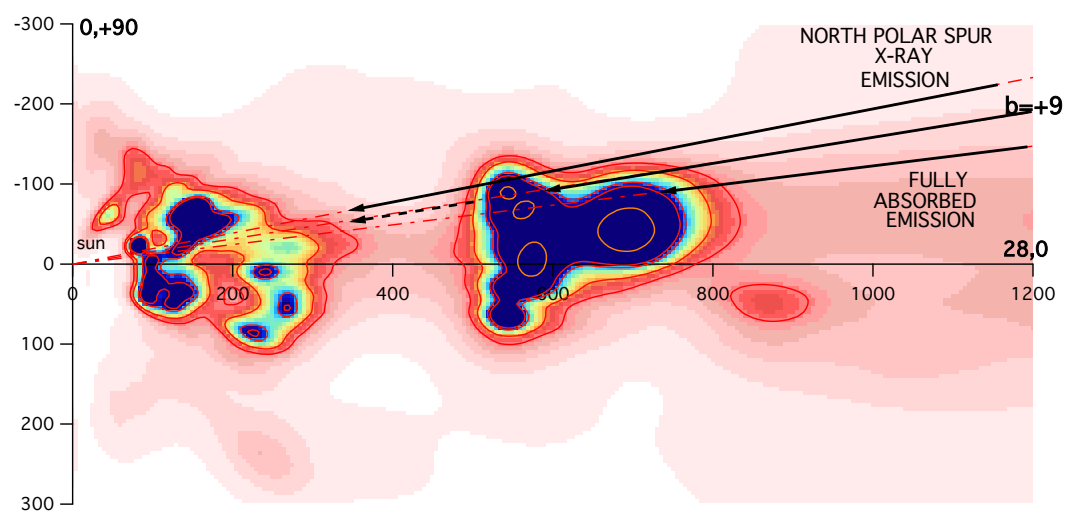

Fig. 9. Differential color excess in a vertical plane containing the Sun and along the Galactic longitude $l=28^{\circ}$. The north Galactic pole is at the top. The map shows the distribution of Aquila Rift clouds and more distant structures. The $b=+9^{\circ}$ dashed line corresponds to the latitude above which X-rays from the North Polar Spur (NPS) start being detected. The very thick cloud absorbing predominantly the NPS at $b \leq+9^{\circ}$ is located between 500 and $800 \mathrm{pc}$.

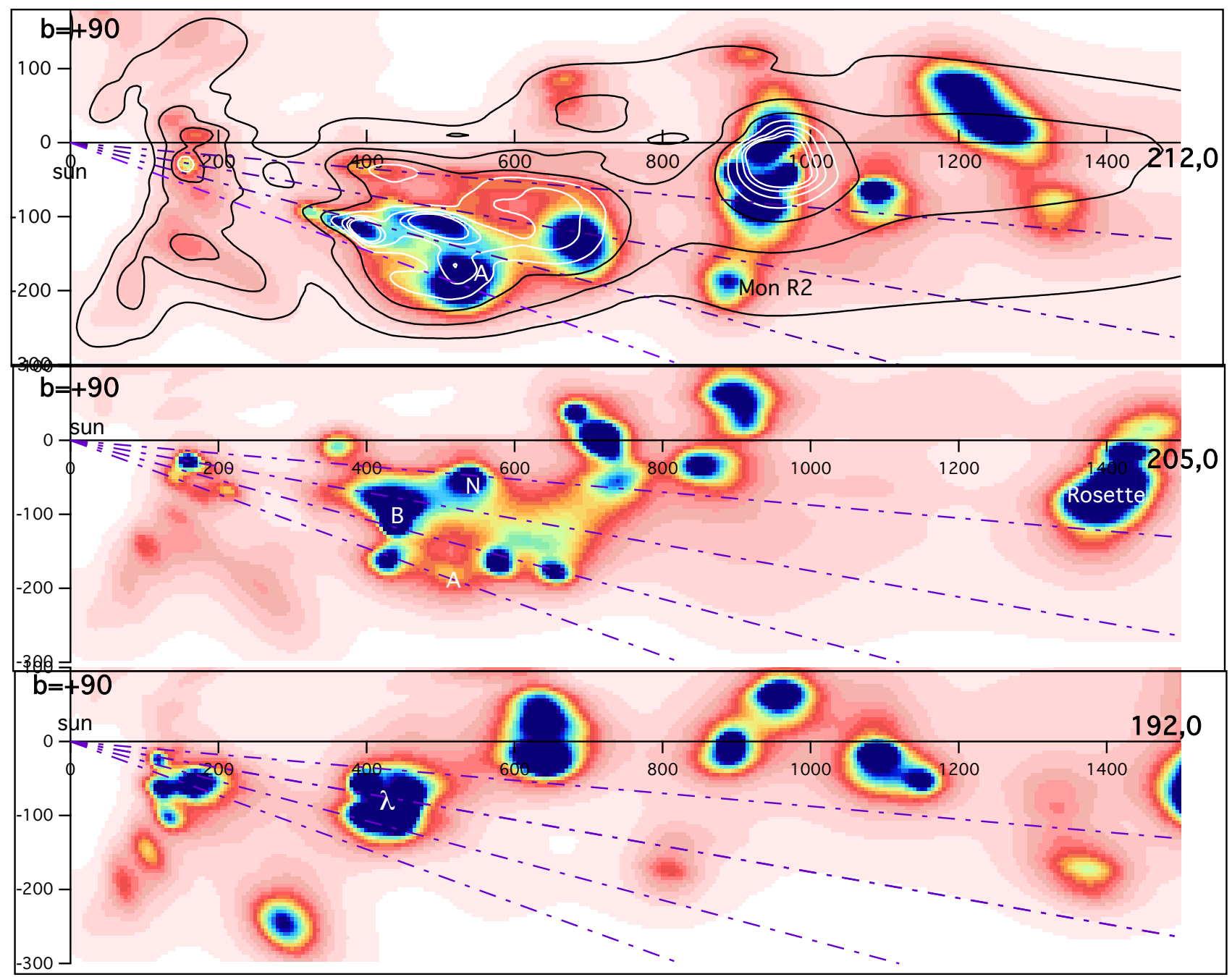

Fig. 10. Differential color excess in a vertical plane containing the Sun and along the Galactic longitudes $1=212,205$, and $192^{\circ}$ from top to bottom. The maps show the distribution of the main Orion clouds. Superimposed as black or white lines on the top graph are isocontours of the differential color excess from the previous inversion of Capitanio et al. (2017). The new 3D distribution reveals more clouds at large distance (e.g., the clouds beyond $800 \mathrm{pc}$ ) and resolves more structures in the main Orion region between 400 and $700 \mathrm{pc}$. We indicate the structures A, B, N as well as $\lambda$ Ori, Mon R2, and Rosette at the locations predicted by Lombardi et al. (2011) and Schlafly et al. (2015).

$1=\sim 60^{\circ}$ and $\sim 240^{\circ}$. It is now well established that the Local Bubble is filled with soft X-ray emitting hot gas, ending years of controversies about its contribution to the soft X-ray diffuse background (Izmodenov et al. 1999; Puspitarini et al. 2014; Galeazzi et al. 2014; Snowden et al. 2015), and that the wide cavity in the third quadrant also contains hot, X-ray emitting gas (Puspitarini et al. 2014). However, it is unclear whether the elongated cavity seen in Fig. 12 at $1=\sim 60-70^{\circ}$ is also filled with hot gas. Additional maps and measurements of the physical state of the IS gas in all cavities and of the cloud motions should help shed light on the source of the peculiar alignment seen in Figs. 7, 11, and 12. 


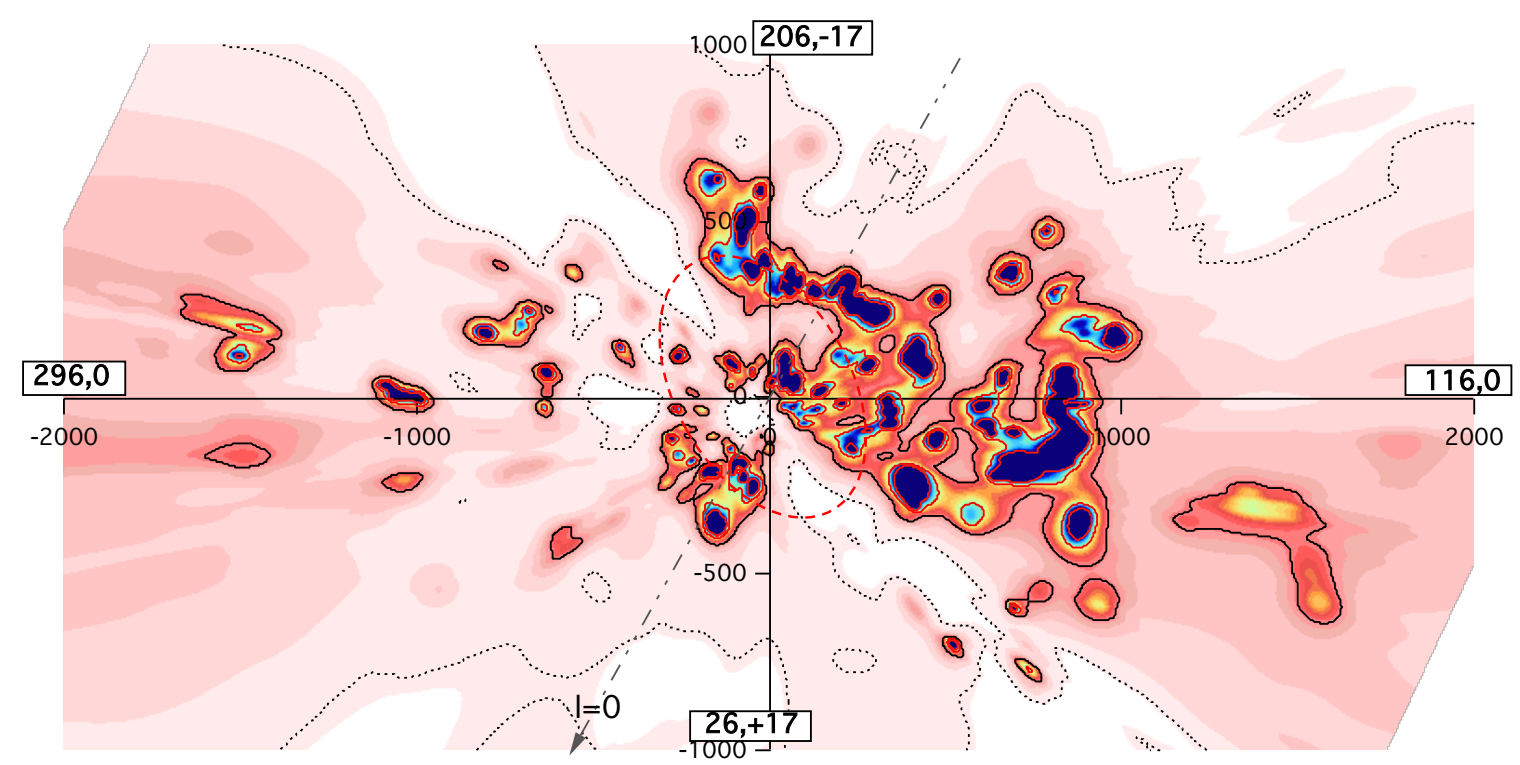

Fig. 11. Planar cut in the 3D distribution of differential color excess for an inclined plane that does not contain the Sun. The image is centered on the location defined by the spherical coordinates $(l, b, d)=\left(180.4^{\circ}, 0^{\circ}, 104 \mathrm{pc}\right)$. The plane is inclined by $17.4^{\circ}$ with respect to the Galactic plane and the longitude of the ascending node is $l=296.1^{\circ}$. It corresponds to the Gould Belt plane defined by Perrot \& Grenier (2003). The units are mag $\mathrm{pc}^{-1}$. The coordinates of the four axes are indicated in white boxes. The ellipse found by Perrot \& Grenier (2003) to best represent Gould belt clouds and motions as an expanding and rotating structure is superimposed (dashed red line). For this plane the boundary of our computational volume is reached at about $1 \mathrm{kpc}$ along the $Y$-axis.

\section{Integration of local maps in the GUMS}

Three-dimensional dust maps of the entire Milky Way are a major ingredient of the Besançon model (Robin et al. 2003) and the Gaia Universe Model Snasphot (GUMS, Robin et al. 2012b,a) which simulates the entire sky as seen by Gaia. GUMS computes the extinction created by an imposed 3D distribution for each of the simulated stars of the Besançon model. Previous versions of GUMS were based on the 3D dust model of Drimmel et al. (2003), and an updated version of the Besançon model ${ }^{4}$ includes the Marshall et al. (2006) maps at low latitudes $\left(-10 \leq b \leq+10^{\circ}\right)$.

As mentioned in the introduction, the Marshall et al. (2006) method used the Besançon model of stellar populations and adjusted a 3D distribution of dust to reproduce the counting statistics on magnitudes and colors from the 2MASS survey. Because it uses star counting and the massive 2MASS survey, the Marshall et al. (2006) method allows a good representation of the extinction in distant and opaque regions. At variance with the Marshall et al. (2006) maps, our local maps described above, which are based on measured absorptions of the light of individual stars, provide the maximum amount of detail within the first few hundred parsecs, and they cover the whole sky. However, due to strong biases in target visibility that are linked to the extinction, they do not reproduce well the most opaque clouds. The two types of information are, in a certain way, complementary, and for this reason we have started to couple the catalog of individual extinctions presented above and the Marshall et al. (2006) model. We note that, at least for this first attempt, we did not aim to achieve the same spatial resolution as in the above inverted maps, but only a more modest resolution of $100 \mathrm{pc}$. The resulting combined model will be used in future GUMS simulations, and comparisons will be made with the previous results; here we only describe the method used to merge the two sets of informa-

\footnotetext{
4 http://model2016.obs-besancon.fr/modele_descrip.php
}

tion, and present it as an additional illustration of the ongoing stellar - interstellar synergy in the context of Gaia.

For such a merging, the Bayesian approach is particularly well adapted. The Marshall et al. (2006) model was taken as the prior distribution close to the Galactic plane $\left( \pm 10^{\circ}\right.$ in latitude), while at higher latitudes we used as prior a dust density that decreases exponentially with the distance to the Plane, has a scale height of $200 \mathrm{pc}$, and is otherwise invariant. Using a scale height above average measurements avoids the loss of tenuous high-latitude structures during the inversion, which may happen when the prior is too low. The information contained in the individual extinctions of the nearby stars was then used to constrain the local distribution, following the formalism described in Vergely et al. (2010) and after replacement at low latitudes of the galactic exponential a priori model with the Marshall model. Because the latter model is available under the form of extinctions at increasing distance intervals for series of radial directions that are independent of each other, we have maintained this approach; we have treated each direction independently and selected, around each of them, those observed targets that fall the closest to the chosen sightline. More specifically, the 3D space is decomposed in cubic cells of $50 \mathrm{pc}$ in size, and for each cell we computed the average of the extinction of all target stars it contains. As a result of this choice, substructures smaller than $100 \mathrm{pc}$ are not solved by this model, as noted above. The standard deviation of the extinction of stars in each cell is used to estimate the variability of the extinction inside the cell and this variability is considered as the error on the extinction. A limitation is made according to the number of targets falling in the cell: when this number is below 3 , the cell is not used. For each direction, we assembled all validated cells crossed by the sightline, and a Bayesian optimization of the cumulative extinction was performed up to $\mathrm{d}=1 \mathrm{kpc}$ or $Z=600 \mathrm{pc}$, whichever was reached first. Beyond this limit the extinction is constrained to increase linearly with distance until it reaches the value of the Marshall model at $1.5 \mathrm{kpc}$. Beyond $1.5 \mathrm{kpc}$ the Marshall model is used. 


\section{R. Lallement et al.: Local ISM 3D maps}

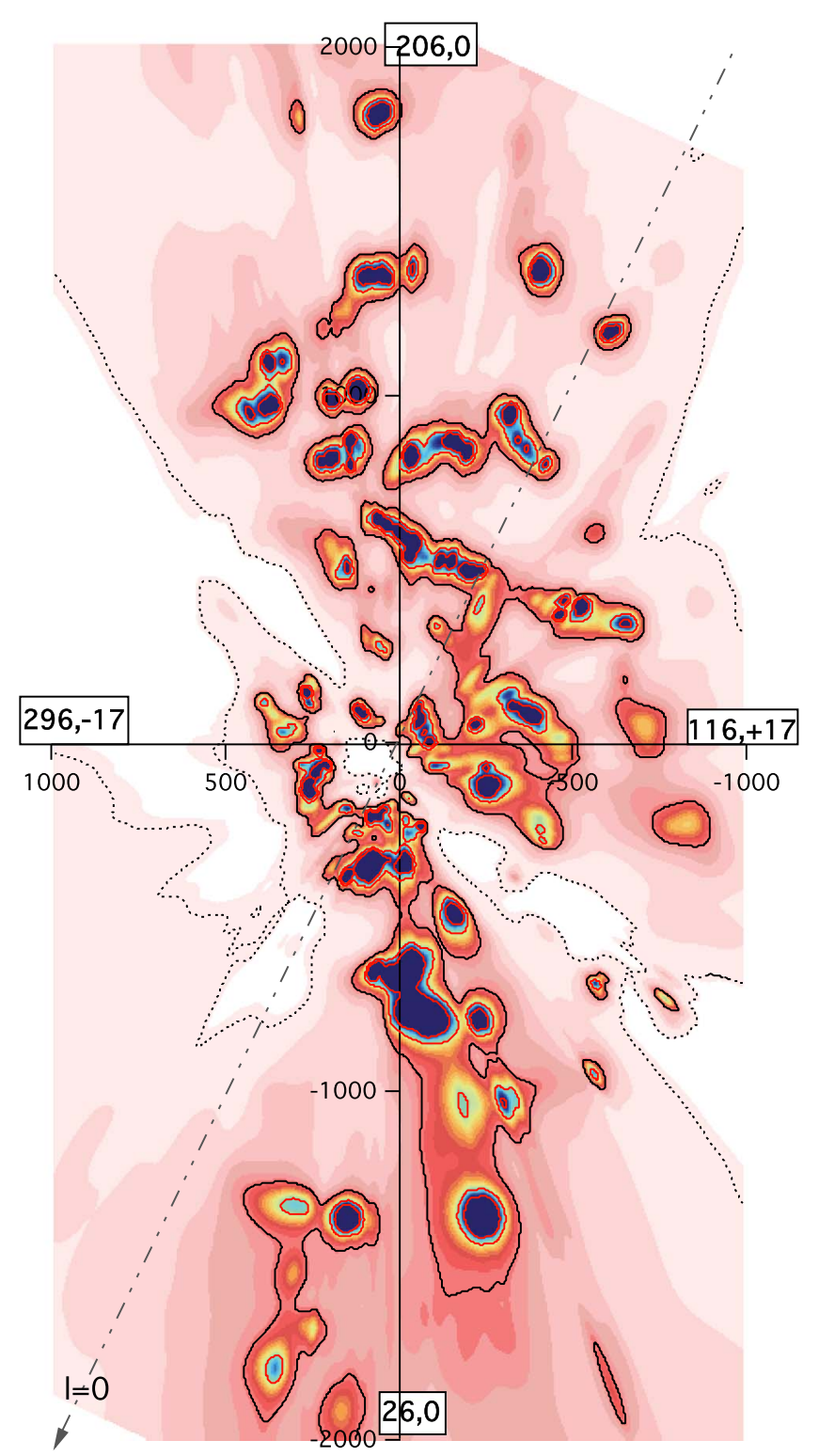

Fig. 12. Same as Fig. 11 after rotation of the plane by $90^{\circ}$ around a vertical axis. The inclination with respect to the Galactic plane and the center of the image are the same. The coordinates of the four axes are indicated in white boxes. Along the $X$-axis the boundary of our computational volume is reached at about $1 \mathrm{kpc}$.

The new GUMS model including the local maps and other improvements will be presented elsewhere by the Besançon group.

\section{Summary and perspectives}

In this work we have illustrated the ongoing synergy between stellar calibrations based on Gaia and ground-based surveys on the one hand, and the construction of 3D maps of the IS dust on the other hand. Three-dimensional maps of the local ISM based on wide- and narrowband photometric determinations of color excesses of individual target stars and TGAS or photometric distances have been used to select non-reddened or weakly reddened targets. These selected targets have been employed to obtain effective temperature-metallicity dependent calibrations of the $\left(G-K_{S}\right)_{0}$ color for APOGEE red giants. These calibrations and subsequent computations of color- and absorption- dependent extinction coefficients in the $G$ band have been used to derive extinction values for most of APOGEE-DR14 red giant targets. Distances were calculated in parallel based on Padova isochrones and the magnitude independent of extinction $K_{J-K_{s}}$.

The new distance-extinction data were added to the database used for the initial 3D dust maps. Doing so, we associated for the first time extinction estimates from ground photometry alone with estimates from both ground data (2MASS, SDSS/APOGEE) and space data (Gaia). A Bayesian inversion of the combined, more than twice as large, extinction catalog was performed. The resulting 3D maps of dust contain a large number of additional structures, especially at distances beyond $400-500 \mathrm{pc}$. To illustrate the improvements, we showed 2D maps of cumulative reddening integrated up to $300 \mathrm{pc}, 800 \mathrm{pc}$, and finally up to the boundary of the $4000 \times 4000 \times 600 \mathrm{pc}^{3}$ computational volume, and we compared them with total reddening maps deduced from emission data. We showed the dust cloud distribution in several planes: (i) all large-scale dust features above $\simeq$ $b=+20^{\circ}$ or below $b=-20^{\circ}$ were found in the $300 \mathrm{pc}$ integrated maps, showing they are local; (ii) in the Galactic plane the addition of more distant clouds starts to reveal a gap between the Local Arm and the Carina-Sagittarius Arm; (iii) in the Aquila rift region, mapping improvements allow us to bring more stringent constraints on the minimum distance to the source of the X-ray bright North Polar Spur, now found to originate beyond 800 pc; (iv) the spatial distribution of the clouds in the Orion region is now much better defined and we were able to identify in the 3D maps the structures found by Lombardi et al. (2011) and Schlafly et al. (2015); (v) in the Gould belt plane inclined with respect to the Galactic plane, the map reveals two distinct cloud complexes separated by a series of cavities instead of an ellipsoidal structure; (vi) in a plane similarly inclined by $17^{\circ}$ but with an orientation that differs by $90^{\circ}$, the Local Arm appears to be made of $a \geq 3 \mathrm{kpc}$ long chain of structures oriented along a $1 \simeq 35-225^{\circ}$ direction, again with a dividing line made of cavities oriented along a $1 \simeq 60-240^{\circ}$ axis. The Local Arm in this plane looks like a double bridge between Carina-Sagittarius and Perseus.

New online tools were made available to use the 3D distribution of $\mathrm{dust}^{5}$. It is possible to draw an image of the dust distribution in any chosen plane, containing the Sun or not. We emphasize that this type of tomographic representation is well adapted to full $3 \mathrm{D}$ inversions, which have the advantage of showing how cloud complexes are related to each other in 3D space. As we already warned in previous similar works, the absolute values of the integrated extinction between the Sun and each point in the computation volume have uncertainties due to the regularization constraints, in particular they may underestimate the extinction in the cases of sightlines crossing cloud cores. This loss of angular resolution is the price to pay for using full 3D correlations between neighboring points. All images and cumulative extinctions can be downloaded, as can the 3D distribution itself.

The productive feedback between 3D dust maps and photometric calibrations we have illustrated here may be developed further, especially in the context of future Gaia data releases. Once 3D maps reliable up to a given distance are obtained, they can be used as prior solutions for the derivation of extinctions towards more distant stars and for additional calibrations. At the same time, Gaia parallaxes with increasing accuracy will become available during the whole mission, allowing us to refine

\footnotetext{
http://stilism.obspm.fr
} 
photometric determinations of the extinction and to constrain more tightly the distances to the absorbing clouds.

Acknowledgements. $\quad$ R. L., J. L. V., C. B., and F. A. acknowledge support from the "Agence Nationale de la Recherche" through the STILISM project (ANR-12-BS05-0016-02). L. C. acknowledges doctoral grant funding from the Centre National d'Etudes Spatiales (CNES). M. E. acknowledges funding from the "Region Ile-de-France" through the DIM-ACAV project. C. D acknowledges post-doctoral grant funding from Centre National detudes Spatiales (CNES) and support from the LabEx P2IO, the French ANR contract 05BLAN-NT09-573739. Funding for the Sloan Digital Sky Survey IV has been provided by the Alfred P. Sloan Foundation, the U.S. Department of Energy Office of Science, and the Participating Institutions. SDSS-IV acknowledges support and resources from the Center for High-Performance Computing at the University of Utah. The SDSS web site is www.sdss.org. SDSS-IV is managed by the Astrophysical Research Consortium for the Participating Institutions of the SDSS Collaboration including the Brazilian Participation Group, the Carnegie Institution for Science, Carnegie Mellon University, the Chilean Participation Group, the French Participation Group, the Harvard-Smithsonian Center for Astrophysics, Instituto de Astrofísica de Canarias, Johns Hopkins University, Kavli Institute for the Physics and Mathematics of the Universe (IPMU)/ University of Tokyo, Lawrence Berkeley National Laboratory, Leibniz Institut für Astrophysik Potsdam (AIP), Max-Planck-Institut für Astronomie (MPIA Heidelberg), Max-Planck-Institut für Astrophysik (MPA Garching), MaxPlanck-Institut für Extraterrestrische Physik (MPE), National Astronomical Observatories of China, New Mexico State University, New York University, University of Notre Dame, Observatário Nacional/MCTI, The Ohio State University, Pennsylvania State University, Shanghai Astronomical Observatory, Unite Kingdom Participation Group, Universidad Nacional Autónoma de México, University of Arizona, University of Colorado Boulder, University of Oxford, University of Portsmouth, University of Utah, University of Virginia, University of Washington, University of Wisconsin, Vanderbilt University, and Yale University. This work has made use of data from the European Space Agency (ESA) mission Gaia (https://www.cosmos.esa.int/gaia), processed by the Gaia Data Processing and Analysis Consortium (DPAC, https://www. cosmos.esa.int/web/gaia/dpac/consortium). Funding for the DPAC has been provided by national institutions, in particular the institutions participating in the Gaia Multilateral Agreement. This publication makes use of data product from the Two Micron All Sky Survey, which is a joint project of the University of Massachusetts and the Infrared Processing and Analysis Center/California Institute of Technology, funded by the National Aeronautics and Space Administration and the National Science Foundation. This research has made use of the SIMBAD database, operated at CDS, Strasbourg, France.

\section{References}

Abolfathi, B., Aguado, D. S., Aguilar, G., et al. 2018, ApJS, 235, 42 Albareti, F. D., Allende Prieto, C., Almeida, A., et al. 2017, ApJS, 233, 25 Arenou, F., Grenon, M., \& Gomez, A. 1992, A\&A, 258, 104

Arenou, F., Luri, X., Babusiaux, C., et al. 2017, A\&A, 599, A50

Bressan, A., Marigo, P., Girardi, L., et al. 2012, MNRAS, 427, 127

Brooks, S., Gelman, A., Jones, G., \& Meng, X.-L. 2011, Handbook of Markov Chain Monte Carlo (Boca Raton: CRC Press)

Capitanio, L., Lallement, R., Vergely, J. L., Elyajouri, M., \& Monreal-Ibero, A. 2017, A\&A, 606, A65

Chabrier, G. 2001, ApJ, 554, 1274

Chen, B. Q., Schultheis, M., Jiang, B. W., et al. 2013, A\&A, 550, A42

Danielski, C., Babusiaux, C., Ruiz-Dern, L., Sartoretti, P., \& Arenou, F. 2018 A\&A, 614, A19
Drimmel, R., Cabrera-Lavers, A., \& López-Corredoira, M. 2003, A\&A, 409, 205 Elyajouri, M., Monreal-Ibero, A., Remy, Q., \& Lallement, R. 2016, ApJS, 225, 19

Gaia Collaboration (Brown, A. G. A., et al.) 2016a, A\&A, 595, A2

Gaia Collaboration (Prusti, T., et al.) 2016b, A\&A, 595, A1

Galeazzi, M., Chiao, M., Collier, M. R., et al. 2014, Nature, 512, 171

García Pérez, A. E., Allende Prieto, C., Holtzman, J. A., et al. 2016, AJ, 151, 144

Green, G. M., Schlafly, E. F., Finkbeiner, D. P., et al. 2015, ApJ, 810, 25

Izmodenov, V. V., Lallement, R., \& Malama, Y. G. 1999, A\&A, 342, L13

Kaltcheva, N. T., Golev, V. K., \& Moran, K. 2014, A\&A, 562, A69

Kos, J., Zwitter, T., Wyse, R., et al. 2014, Science, 345, 791

Lallement, R., Vergely, J.-L., Valette, B., et al. 2014, A\&A, 561, A91

Lallement, R., Vergely, J.-L., Puspitarini, L., et al. 2015, Mem. Soc. Astron. It., 86,626

Lallement, R., Snowden, S., Kuntz, K. D., et al. 2016, A\&A, 595, A131

Liu, C., Bailer-Jones, C. A. L., Sordo, R., et al. 2012, MNRAS, 426, 2463

Lombardi, M., Alves, J., \& Lada, C. J. 2011, A\&A, 535, A16

Majewski, S. R., Schiavon, R. P., Frinchaboy, P. M., et al. 2017, AJ, 154, 94

Marrese, P. M., Marinoni, S., Fabrizio, M., \& Giuffrida, G. 2017, A\&A, 607, A105

Marshall, D. J., Robin, A. C., Reylé, C., Schultheis, M., \& Picaud, S. 2006, A\&A, 453, 635

Perrot, C. A., \& Grenier, I. A. 2003, A\&A, 404, 519

Planck Collaboration Int. XIX. 2015, A\&A, 576, A104

Plummer, M. 2003, JAGS: A program for analysis of Bayesian graphical models using Gibbs sampling

Puspitarini, L., \& Lallement, R. 2012, A\&A, 545, A21

Puspitarini, L., Lallement, R., Vergely, J.-L., \& Snowden, S. L. 2014, A\&A, 566, A13

Queiroz, A. B. A., Anders, F., Santiago, B. X., et al. 2018, MNRAS, 476, 2556

Rezaei Kh., S., Bailer-Jones, C. A. L., Hanson, R. J., \& Fouesneau, M. 2017, A\&A, 598, A125

Robin, A. C., Reylé, C., Derrière, S., \& Picaud, S. 2003, A\&A, 409, 523

Robin, A. C., Luri, X., Reylé, C., et al. 2012a, VizieR Online Data Catalog: $\mathrm{VI} / 137$

Robin, A. C., Luri, X., Reylé, C., et al. 2012b, A\&A, 543, A100

Rodrigues, T. S., Girardi, L., Miglio, A., et al. 2014, MNRAS, 445, 2758

Ruiz-Dern, L., Babusiaux, C., Arenou, F., Turon, C., \& Lallement, R. 2018, A\&A, 609, A116

Sale, S. E. \& Magorrian, J. 2014, MNRAS, 445, 256

Sale, S. E. \& Magorrian, J. 2015, MNRAS, 448, 1738

Sale, S. E. \& Magorrian, J. 2018, MNRAS, in press, DOI: $10.1093 / \mathrm{mnras} /$ sty 2118

Sale, S. E., Drew, J. E., Barentsen, G., et al. 2014, MNRAS, 443, 2907 Santiago, B. X., Brauer, D. E., Anders, F., et al. 2016, A\&A, 585, A42 Schlafly, E. F., Green, G., Finkbeiner, D. P., et al. 2015, ApJ, 799, 116 Schlegel, D. J., Finkbeiner, D. P., \& Davis, M. 1998, ApJ, 500, 525 Schultheis, M., Chen, B. Q., Jiang, B. W., et al. 2014a, A\&A, 566, A120 Schultheis, M., Zasowski, G., Allende Prieto, C., et al. 2014b, AJ, 148, 24 Skrutskie, M. F., Cutri, R. M., Stiening, R., et al. 2006, AJ, 131, 1163 Snowden, S. L., Heiles, C., Koutroumpa, D., et al. 2015, ApJ, 806, 119 Sofue, Y., Habe, A., Kataoka, J., et al. 2016, MNRAS, 459, 108

Sun, X. H., Landecker, T. L., Gaensler, B. M., et al. 2015, ApJ, 811, 40 Tarantola, A. \& Valette, B. 1982, Rev. Geophys. Space Phys., 20, 219 van Loon, J. T., Bailey, M., Tatton, B. L., et al. 2013, A\&A, 550, A108

Vergely, J.-L., Freire Ferrero, R., Siebert, A., \& Valette, B. 2001, A\&A, 366, 1016 Vergely, J.-L., Valette, B., Lallement, R., \& Raimond, S. 2010, A\&A, 518, A31 Wang, J., Shi, J., Pan, K., et al. 2016, MNRAS, 460, 3179

Welsh, B. Y., Lallement, R., Vergely, J.-L., \& Raimond, S. 2010, A\&A, 510, A54

Zasowski, G., Ménard, B., Bizyaev, D., et al. 2015, ApJ, 798, 35 